\section{Violencia y salud mental asociados a pensar o haber intentado emigrar internacionalmente por adolescentes mexicanos}

\author{
Violence and mental health issues among Mexican \\ adolescents that have considered or attempted \\ cross-border migration
}

\section{Violência e problemas de saúde mental entre adolescentes mexicanos que têm considerado ou tentado migrar internacionalmente}

Ruben Chavez-Ayala 1

Emanuel Orozco-Núñez 1

Marcela Sánchez-Estrada 2

Carlos Hernández-Girón 3

doi: 10.1590/0102-311X00119516

\title{
Resumen
}

El objetivo del presente estudio fue estimar la contribución de ser víctima de la violencia con pensar o haber intentado emigrar a los Estados Unidos de América por adolescentes mexicanos, con la intervención de variables de salud mental (autoestima emocional, autoestima escolar, depresión, ideación suicida e intento suicida), como mediadoras de los efectos. Este estudio utiliza diseño transversal, con muestra estratificada por conglomerados de 13.198 adolescentes de la Segunda Encuesta Nacional sobre Exclusión, Intolerancia y Violencia en escuelas públicas de México en 2009. El análisis se realizó mediante modelos de regresión propuestos por Baron \& Kenny. La prevalencia de pensar o haber intentado emigrar fue de 23,1\%. El promedio de edad fue de 16,36 años. El 54,9\% fueron mujeres. El 56\% de los encuestados fueron de clase baja. Las variables de salud mental que parcialmente actuaron como mediadoras fueron ideación suicida (35,9\%), depresión (19,2\%), intento de suicidio (17,7\%), autoestima emocional (6,2\%) y autoestima escolar (3,4\%), por la violencia moderada en la familia, y autoestima emocional $(17,5 \%)$ por rechazo social en la escuela e ideación suicida $(8,1 \%)$ por daños materiales en la escuela. Se encontró mayor impacto de mediadoras en mujeres que en hombres en el pensar o haber intentado emigrar. Se discute la importancia de incorporar la prevención de la violencia en los contextos sociales investigados e incorporar a la salud mental en la atención a la violencia en adolescentes y en los programas de salud pública en zonas de tránsito de migrantes ilegales.

Violencia Doméstica; Salud Mental; Emigración e Inmigración; Adolescente

\author{
Correspondencia \\ M. Sánchez-Estrada \\ Secretaría Académica, Instituto Nacional de Salud Pública. \\ Av. Universidad 655, Sta. Ma. Ahuacatitlan, Cuernavaca / \\ Morelos - 62100, México. \\ rchavez@insp.mx \\ 1 Centro de Investigaciones en Sistemas de Salud, Instituto \\ Nacional de Salud Pública, Cuernavaca, México. \\ 2 Secretaría Académica, Instituto Nacional de Salud Pública, \\ Cuernavaca, México. \\ 3 Centro de Investigaciones en Salud Pública, Instituto Nacional \\ de Salud Pública, Cuernavaca, México.
}




\section{Introducción}

La Organización Mundial de la Salud (OMS) 1 (p. 5) define la violencia como: "El uso deliberado de la fuerza física o el poder, ya sea en grado de amenaza o efectivo contra uno mismo, otra persona, o contra un grupo o comunidad, que cause o tenga muchas probabilidades de causar lesiones, muerte, daños psicológicos, trastornos del desarrollo o privaciones". Según la Organización de las Naciones Unidas (ONU) 2, en América Latina, la violencia está presente en la socialización temprana de los adolescentes y los niños. El castigo corporal es aceptado en la crianza como una forma de disciplina no identificada socialmente como violencia, la cual tiene efectos en el desarrollo normal de los menores, y sus consecuencias dependen del tipo y la intensidad de la violencia 3,4, asociada con el desarrollo de trastornos emocionales 5, síntomas físicos y problemas de salud 6, drogadicción y alcoholismo 7,8, el desarrollo de trastorno limítrofe de personalidad 9 y abandono del hogar 10 . La violencia doméstica incluye también victimización y exposición a violencia entre padres, lo que conduce a comportamientos disruptivos, pobre rendimiento académico 11 , ideación suicida 12 y huida del hogar 10 . Pero también la violencia en la escuela y la comunidad afectan cognitiva, emocionalmente y comportamentalmente a los menores 4,13, dando lugar a numerosos riesgos involucrados en la búsqueda de estrategias desviantes de supervivencia 13,14, incluida la migración internacional, perturbando el desarrollo educativo de los jóvenes y el plan de vida 5,15. Según la Organización para la Cooperación y el Desarrollo Económicos (OCDE), de los 33 países estudiados, México en ambiente escolar ocupa el primer lugar en lesiones físicas ocasionadas por la violencia, vandalismo escolar y robo, tercero en el uso y posesión de drogas, y cuarto en intimidación o abuso verbal entre estudiantes 16 . Como se ha reportado el bullying escolar está asociado con depresión, ansiedad y trastorno de estrés posttraumático 17; bajo ajuste social, estrés psicológico y somatización física 18; baja autoestima emocional, temor a victimización en la escuela, y bajo rendimiento escolar. Esto, a su vez, podría provocar el fracaso y el abandono escolar 2,19.

Con respecto a la violencia en el barrio en México, el crimen parece ir en aumento. Entre 2010 y 2011, las denuncias de adolescentes víctimas de amenazas, lesiones, robo y daños a la propiedad crecieron, y se informa que la principal causa de muerte por violencia en el grupo de 10 a 14 años es por el uso de armas de fuego y otras armas; y en el grupo de 15 a 19 años, la muerte por violencia es la segunda causa de muerte en varones y la tercera en mujeres 20 .

Se ha informado que la migración internacional de niños y jóvenes es también una de las consecuencias de la violencia familiar y social que existe en América Latina y el Caribe, que ocupa el segundo lugar como la región que expulsa más migrantes en el mundo. En ella, el 25\% de migrantes son menores de 20 años de edad. Según la Oficina del Alto Comisionado de las Naciones Unidas para los Refugiados (ACNUR), una proporción significativa del total de adolescentes y jóvenes migrantes se traslada a los EE.UU., principalmente para escapar de la violencia 21 ; durante el viaje, están expuestos a diferentes formas de victimización en rutas de migración ilegal, controladas por cárteles y pandillas involucradas en el tráfico de drogas, armas y personas 22,23. Además, están expuestos a comportamientos de riesgos para la salud, lo que puede conducir a conductas como el uso de sustancias ilegales y alcohol 24,25, trabajar en la prostitución 26 , tener relaciones sexuales sin protección con riesgo de infección por VIH 27, y con riesgo de desarrollo de trastornos emocionales 28.

La migración internacional ha sido tradicionalmente asociada con los ciclos económicos y las disparidades en el desarrollo estructural y el empleo 29,30; y se han realizado pocos estudios sobre la contribución de la violencia como factor de riesgo de emigrar internacionalmente en la población adolescente, y esto es preocupante, ya que de acuerdo con el censo de población de 2010, entre 2005 a 2010, el 24,6\% de los migrantes mexicanos tenían menos de 19 años de edad 31 .

En base a lo anterior, sin muchas investigaciones sobre este tema, el objetivo del presente estudio fue estimar la contribución de la exposición a la violencia en el hogar, el bullying y el ambiente violento escolar y violencia en el barrio con pensar o haber intentado emigrar a los EE.UU. por los adolescentes mexicanos, con la intervención de variables de salud mental como la autoestima emocional y autoestima escolar, depresión y la ideación y el intento suicida, como mediadoras del efecto, para identificar los contextos sociales de riesgo y las formas de violencia, a fin de planificar estrategias y políticas de salud pública para la prevención de la migración internacional indocumentada de los adolescentes. 


\section{Metodología}

\section{Datos}

La información, proveniente de la Segunda Encuesta Nacional sobre Exclusión, Intolerancia y Violencia en las Escuelas de Educación Media Superior, realizada en 2009, en la República Mexicana. Se consideró un diseño muestral probabilístico, estratificado y por conglomerados. Para asegurar un tamaño de muestra aceptable, se consideró como indicador la prevalencia de abuso sexual (6\%), reportada en investigaciones en estudiantes mexicanos. Para el cálculo del tamaño de muestra se consideró: la proporción más pequeña a estimar $\mathrm{p}=0,05$; nivel de confianza del $95 \%\left(\mathrm{Z}_{\alpha / 2}\right)$; error relativo máximo $(\mathrm{r})$ dispuesto a aceptar de 20\%; efecto de diseño esperado (deff) de 1,7 y un factor de corrección por tasa de no respuesta (TR) del 15\%.

La muestra se tomó en dos etapas a partir de un marco muestral de todas las escuelas de nivel medio superior registradas por la Secretaría de Educación Pública. En la primera etapa, de 4.463 escuelas se seleccionaron 105 de ellas, y en la segunda, tres grupos de estudiantes se escogieron aleatoriamente en cada escuela, uno por cada grado escolar. Se logró la participación de 16.700 adolescentes de los cuáles se obtuvieron 14.200 encuestas (85\%).

\section{Material y métodos}

La aplicación de esta encuesta fue realizada por personal capacitado, quien viajó a todos los estados de la República Mexicana. Se pidió el consentimiento de los padres cuando los participantes tenían menos de 18 años de edad, además los propios estudiantes firmaron la carta de consentimiento libre e informado. Estos recibieron información sobre el contenido y la realización del cuestionario auto-aplicado, y los datos se recogieron durante el horario escolar en el aula de cada grupo escolar. El estudio siguió los principios de la Declaración de Helsinki y fue aprobado por el comité de ética del Instituto Nacional de Salud Pública de México.

\section{Medidas}

\section{- Variable de resultado}

(a) Pensar o haber intentado emigrar a los EE.UU.: esta variable se midió a través de la pregunta “ ¿Has pensado o intentado irte a trabajar a Estados Unidos?” con las opciones de respuesta de (0) no y (1) sí.

\section{- Las variables independientes}

(a) Índice de nivel socioeconómico: se midió usando un índice de nivel socioeconómico 32 construido por la combinación de las siguientes variables: nivel de hacinamiento, material del piso de la vivienda, disponibilidad de agua potable, forma de eliminación de excretas, escolaridad del jefe de familia y número de bienes en la vivienda. Las categorías del índice fueron: (1) bajo, (2) medio y (3) alto.

(b) Nivel de escolaridad de los padres: esta variable se construyó a partir del máximo nivel de escolaridad alcanzado por cada padre de familia. Se agruparon como (1) escolaridad baja: sin escolaridad, primaria incompleta y completa; (2) regular: secundaria incompleta, completa y preparatoria incompleta; (3) alta: preparatoria completa, licenciatura incompleta y completa, hasta posgrado incompleto y completo. Para el análisis multivariado se utilizó al grupo alto como referencia, de acuerdo a datos del censo nacional de México del 2010 que señala el promedio nacional en el tercer año de secundaria. (c) Víctima de la violencia en el hogar: para medir esta variable se utilizaron reactivos de la escala de tácticas de conflictos de padres a hijos de Straus et al. 33, previamente utilizados en México en población escolar adolescente. Esta variable contiene dos factores de maltrato que se diferencian del castigo corporal culturalmente legítimo, pasando a ser agresión criminal por el grado de peligrosidad, como potenciales generadores de lesiones físicas y daño emocional. Uno llamado de violencia moderada y el otro de violencia severa. El primero con seis ítems y el segundo con siete. Todos precedidos por la frase “¿Alguna vez en tu vida algún familiar (padre, madre, hermanos, tíos, etc.)?”. Los reactivos se evaluaron 
con la escala: (1) nunca, (2) pocas veces, (3) regularmente y (4) muchas veces. Posteriormente, se promediaron los ítems para crear una variable continua por cada dimensión.

(d) Índice de violencia entre sus padres: para esta variable se utilizaron cuatro reactivos por cada padre provenientes de la escala de Straus et al. ${ }^{33}$. Una escala de violencia del padre hacia la madre y otra de la madre hacia el padre, cada una de cuatro ítems, precedida por la siguiente frase: "Cuándo tus padres discuten, la reacción de tu padre/madre hacia tu madre/padre es". Los actos fueron: le insulta, le grita, le tira objetos o le golpea con ellos, y le empuja o le pega; los ítems fueron evaluados con la escala: (1) nunca, (2) casi nunca, (3) algunas veces y (4) muchas veces. Posteriormente, se promediaron los ítems para crear una variable continua.

(e) Víctima de bullying: esta variable se midió mediante el cuestionario Evaluación de la Violencia entre Pares en la Escuela y el Ocio que ha sido validado 34. Cada ítem comienza con la frase "durante los últimos 12 meses, mis compañeros", seguido por una lista de diversos actos violentos. El instrumento contiene tres dimensiones: amenazas, con cinco ítems; rechazo social, con tres ítems y daños a la propiedad, con tres ítems. Se evaluó en una escala: (1) nunca, (2) a veces y (3) a menudo. Posteriormente, se promediaron los ítems para crear una variable continua.

(f) Índice de peligro en la escuela: esta variable se midió mediante el cuestionario validado de Evaluación de la Violencia entre Pares en la Escuela y el Ocio 34 y en esta sección explora la percepción de peligro en el entorno escolar por presencia de pandillas, violencia y venta de droga. Utiliza cuatro ítems precedidos por la frase “en mi escuela...”. Se evaluó con la escala: (1) nunca, (2) pocas veces, (3) a veces, y (4) muchas veces. Posteriormente, se promediaron los ítems para crear una variable continua.

(g) Índice de violencia en el barrio: para medir esta variable se utilizó el cuestionario validado de Evaluación de la Violencia entre Pares en la Escuela y el Ocio ${ }^{34}$. Esta variable explora la percepción de peligro en el barrio por presencia de violencia, pandillas y personas alcohólicas. Se evaluó en la escala: (1) nunca, (2) pocas veces, (3) algunas veces, y (4) siempre. Posteriormente, se promediaron los ítems para crear una variable continua.

\section{- Las variables mediadoras}

(a) Autoestima emocional y la autoestima escolar: estas variables se midieron por la Escala de Autoconcepto Forma A (AFA) 35. La primera variable representa la autoestima emocional percepción de la persona de su estado emocional y relación con otros, evaluada con cinco reactivos. La segunda evalúa autoestima escolar como la visión de logros y capacidades en la escuela medida con cuatro ítems 36 . Se usaron las siguientes opciones de respuesta: (1) nunca, (2) raramente, (3) algunas veces, (4) a menudo y (5) siempre. Posteriormente, se promediaron los ítems para crear una variable continua.

(b) Depresión: para esta variable se utilizó la Escala de Depresión del Centro para Estudios Epidemiológicos (CES-D) 37, la cual ha sido validada en población mexicana. Este instrumento fue evaluado con una escala: (1) nunca, (2) a veces, (3) muchas veces y (4) siempre. Posteriormente, se promediaron los ítems para crear una variable continua.

(c) Ideación e intento suicida: estas dos variables se midieron separadamente, utilizando preguntas tomadas de la Encuesta Nacional de Adicciones de México 38 , considerando la ideación suicida como una respuesta positiva a las preguntas: “ ¿has tenido la sensación de que no vale la pena vivir?”, “ ¿has vivido situaciones ante las que has deseado dejar de existir?” y “'has pensado que vale más morir que vivir?”. Se construyó un índice considerando 0 si no se contestó "sí” a cualquier pregunta, y 3 si la respuesta fue "sí” en las tres; y el intento de suicidio se evaluó con dos preguntas: “ ¿has intentado quitarte la vida?” " “ite has herido, cortado, intoxicado o hecho daño con el fin de quitarte la vida?”. Las categorías de respuesta fueron (1) sí y (0) no, una suma que el procedimiento fue realizado para crear la medida compuesta.

\section{Plan de análisis de datos}

Todos los análisis realizados consideraron el efecto de diseño muestral por conglomerados, incluyendo tipo de unidad de análisis, ponderador y estrato. Se realizó un análisis descriptivo, utilizando frecuencias y medias. Un análisis bivariado para determinar la asociación entre pensar o intentar emigrar a los EE.UU. y las variables independientes con pruebas de chi-cuadrado o t de Student. El análisis de mediación fue realizado en dos fases, siguiendo la metodología propuesta por Baron 
\& Kenny 39 y, posteriormente, por Kenny ${ }^{40}$, quiénes recomiendan el uso del método de regresión como estrategia para la estimación de las mediaciones a través de cinco pasos. Este análisis se realizó para el total de adolescentes y por cada sexo. Paso 1: modelo de regresión logística entre variables de exposición a violencia y variable respuesta de pensar o intentar emigrar. Paso 2: modelos de regresión lineal con variables de exposición y variables mediadoras para mostrar asociación. Paso 3: análisis de regresión logística bivariada entre cada variable mediadora y la variable respuesta. Paso 4: modelos bivariados de regresión logística entre cada variable mediadora y la variable respuesta. Paso 5: se repite modelo de regresión del paso 1, incorporando alternadamente cada variable mediadora para observar cambios en coeficientes de variables de exposición a violencia significativas en el modelo del paso 1. Una variable para ser mediadora debe disminuir el coeficiente de regresión de otras variables de exposición (mediación parcial) o puede reducirlo a cero (mediación total). Para toda la muestra estos modelos fueron ajustados por edad, sexo, nivel socioeconómico, nivel de escolaridad del padre y la madre. Por sexo, se controlaron por las variables sociodemográficas restantes. En la segunda etapa se calculó el porcentaje de efectos directos e indirectos y la realización de test Sobol para estimar la significancia estadística de los porcentajes estimados. Los análisis se completaron mediante el programa Stata, versión 13 (StataCorp LP, College Station, EE.UU.).

\section{Resultados}

El presente análisis incluyó a 13,198 adolescentes de una muestra nacional de estudiantes de escuelas públicas de nivel profesional técnico, bachillerato y bachillerato profesional técnico de México, entre los $14 \mathrm{y}$ los 19 años de edad, que tuvieron cuestionarios completos, con un porcentaje de pérdida del 6,6\%. En esta muestra, la prevalencia de pensar o haber intentado emigrar a los EE.UU. fue de 23,1\%.

Las características sociodemográficas generales de la muestra señalan un promedio de edad de $16,3$ años ( $\mathrm{DE}=1.06)$, con $54,9 \%$ de mujeres, una mayoría de estudiantes de clase baja (56\%), y $36,4 \%$ de las madres, y 33,2\% de los padres habían terminado la escuela primaria como nivel máximo (Tabla 1).

Por variable de pensar o haber intentado emigrar, se observa que el grupo en dicha condición tiene mayor edad $(\mathrm{x}=16,55)$, con mayor porcentaje de hombres (57\%), mayor proporción de estudios terminados en sistema educativo de tres años (26,42\%), nivel socioeconómico bajo $(59,3 \%)$, y madre y padre con niveles educativos bajos ( $42,82 \%$ y $38,22 \%$, respectivamente) (Tabla 1 ).

Se observa que los niveles de exposición a la violencia son en promedio mayores en el grupo que piensa o ha intentado emigrar, en violencia moderada y severa, amenazas, daños a la propiedad, rechazo social e índice de peligro en el barrio. No así en violencia entre los padres (Tabla 2).

Se encontraron diferencias por sexo en victimización por violencias y variables mediadoras, con mayor nivel en mujeres por violencia moderada y exposición a violencia entre padres. En hombres mayor victimización por violencia severa en hogar y mayor victimización en escuela y barrio. En toda variable mediadora las mujeres presentan niveles más altos (Tabla 3).

Con respecto a las asociaciones entre las variables mediadoras y pensar o haber intentado emigrar, todos estuvieron estadísticamente asociados: autoestima emocional $\beta=0,02(0,01 ; 0,04)$; autoestima escolar $\beta=-0,06(-0,08 ;-0,03)$; depresión $\beta=0,09(0,05 ; 0,13)$; ideación suicida $\beta=0,24(0,21 ; 0,27)$; e intento suicida $\beta=0,06(0,04 ; 0,08)$.

En general el riesgo de pensar o haber intentado emigrar es mayor por cada año de incremento en la edad, ser hombre, y tener padres con nivel educativo bajo. También, por cada unidad de incremento en los niveles de violencia moderada en hogar, daños a la propiedad y rechazo social en la escuela y violencia en el barrio. Al comparar por sexo, se observan diferencias en riesgo sólo en la mujer por violencia en la escuela y en hombre por violencia en barrio y nivel de escolaridad baja en la madre (Tabla 4).

La Tabla 5 muestra la contribución de variables mediadoras al pensar o haber intentado emigrar por violencias asociadas. En total el porcentaje más alto fue para ideación suicida $(35,9 \%)$, seguida por depresión (19,2\%), intento suicida (17,7\%), autoestima emocional (6,2\%) y autoestima escolar $(3,42 \%)$, asociadas con violencia moderada en el hogar. La autoestima emocional $(17,5 \%)$ estuvo asociada también con el rechazo social, y la ideación suicida $(8,1 \%)$ con daños a la propiedad. Se observa en mujeres 


\section{Tabla 1}

Características de los estudiantes que piensan o han intentado emigrar a los Estados Unidos. México, 2009.

\begin{tabular}{|c|c|c|c|}
\hline Características & $\begin{array}{l}\text { Total } \\
(\mathrm{N}=13.198) \\
\text { \% o media }\end{array}$ & $\begin{array}{l}\text { No piensan o no han intentado emigrar } \\
\qquad \begin{array}{l}(n=10.150) \\
\% \text { o media }\end{array}\end{array}$ & $\begin{array}{l}\text { Piensan emigrar o lo han intentado } \\
\qquad(\mathrm{n}=3.048) \\
\% \text { o media }\end{array}$ \\
\hline Edad * & 16,36 & 16,29 & 16,55 \\
\hline \multicolumn{4}{|l|}{ Sexo * } \\
\hline Mujer & 54,9 & 58,5 & 42,9 \\
\hline Hombre & 45,0 & 41,4 & 57,0 \\
\hline \multicolumn{4}{|c|}{ Nivel de bachillerato * } \\
\hline 1 & 34,3 & 27,3 & 7,0 \\
\hline 2 & 26,4 & 20,0 & 6,3 \\
\hline 3 & 29,0 & 21,1 & 26,4 \\
\hline 4 & 3,3 & 2,8 & 0,4 \\
\hline 5 & 5,2 & 4,0 & 1,2 \\
\hline 6 & 1,5 & 1,3 & 0,1 \\
\hline \multicolumn{4}{|c|}{ Nivel socioeconómico ** } \\
\hline Clase baja & 56,0 & 54,9 & 59,3 \\
\hline Clase media & 30,6 & 31,2 & 28,6 \\
\hline Clase alta & 13,3 & 13,7 & 12,0 \\
\hline \multicolumn{4}{|c|}{ Nivel de educación de la madre * } \\
\hline Bajo & 36,4 & 34,5 & 42,8 \\
\hline Mediano & 35,6 & 36,0 & 34,5 \\
\hline Alto & 27,8 & 29,4 & 22,7 \\
\hline \multicolumn{4}{|c|}{ Nivel de educación del padre * } \\
\hline Bajo & 33,2 & 24,4 & 38,2 \\
\hline Mediano & 35,3 & 34,9 & 35,4 \\
\hline Alto & 31,3 & 32,7 & 26,8 \\
\hline
\end{tabular}

* $\mathrm{p}<0,001$

** $\mathrm{p}<0,05$.

Nota: prevalencias (\%) y medias ponderadas, de acuerdo con el diseño por conglomerados del estudio.

porcentajes mayores de impacto de mediadoras que en hombres. Se muestran cambios en las razones de momios del modelo de regresión final por presencia de cada variable mediadora en comparación con el modelo inicial.

\section{Discusión}

Según los resultados encontrados, el $23,1 \%$ de esta muestra piensa o han intentado emigrar a los EE.UU. Otras encuestas 41, señalan hasta un 30\% la prevalencia de intención de migrar de adolescentes y en algunos estados de la república es aún mayor, debido a tradiciones familiares migrantes 15 El censo de población mexicana 2010 31, indica que el porcentaje real de migrantes internacionales mexicanos menores de 19 años es de $24,6 \%$.

Una de las causas de este fenómeno, y que se refleja en los datos sociodemográficos del presente estudio, es el bajo nivel socioeconómico de quiénes han pensado o intentado emigrar en un país donde la proporción de adolescentes ha aumentado en 23,88\% en los últimos 25 años y representa el 19,55\% de la población total, con mayor competencia por el empleo. Estas prevalencias muestran que el problema de la migración de adolescentes en México es grave y un área de oportunidad para la investigación y realización de intervenciones. 


\section{Tabla 2}

Las diferencias en la violencia vivida en el entorno familiar, la escuela y el vecindario.

\begin{tabular}{|c|c|c|c|}
\hline & $\begin{array}{l}\text { Total } \\
(\mathrm{N}=13.198) \\
\text { Media }\end{array}$ & $\begin{array}{l}\text { No piensan o no han intentado emigrar } \\
\qquad \begin{array}{c}(\mathrm{n}=10.150) \\
\text { Media }\end{array}\end{array}$ & $\begin{array}{l}\text { Piensan o han intentado emigrar } \\
\qquad(\mathrm{n}=3.048) \\
\text { Media }\end{array}$ \\
\hline \multicolumn{4}{|l|}{ Entorno familiar } \\
\hline \multicolumn{4}{|l|}{ Víctima de violencia en el hogar } \\
\hline \multicolumn{4}{|l|}{ Violencia } \\
\hline Violencia moderada * & 1,45 & 1,41 & 1,56 \\
\hline Violencia severa * & 1,03 & 1,02 & 1,05 \\
\hline $\begin{array}{l}\text { Índice de violencia del padre contra } \\
\text { la madre * }\end{array}$ & 1,66 & 1,76 & 1,54 \\
\hline $\begin{array}{l}\text { Índice de violencia de la madre contra } \\
\text { el padre ** }\end{array}$ & 1,63 & 1,72 & 1,53 \\
\hline \multicolumn{4}{|l|}{ Ambiente escolar } \\
\hline \multicolumn{4}{|l|}{ Víctima de bullying } \\
\hline Amenazas * & 1,03 & 1,03 & 1,06 \\
\hline Daños a la propiedad * & 1,23 & 1,21 & 1,31 \\
\hline Rechazo social * & 1,30 & 1,28 & 1,36 \\
\hline Índice de peligro en la escuela $* \star \star$ & 1,67 & 1,66 & 1,70 \\
\hline \multicolumn{4}{|l|}{ Vecindario } \\
\hline Índice de violencia en el barrio * & 2,31 & 2,27 & 2,44 \\
\hline
\end{tabular}

Comparación en las violencias vividas: * $p<0,001 ; * * p<0,01 ; y * * * p<0,05$.

Nota: medias ponderadas, de acuerdo con el diseño por conglomerados.

Por otra parte, los resultados más importantes de este estudio señalan que algunas formas de violencia se asocian con pensar o haber intentado emigrar a los EE.UU., y que los adolescentes de nivel medio superior que han sufrido violencia en el hogar y bullying escolar tienen más trastornos psicológicos que incrementan la probabilidad de dichas acciones.

En cuanto al contexto familiar, todas las variables de salud mental fueron mediadoras por violencia moderada, siendo la depresión, ideación e intento suicidas las que tuvieron mayor porcentaje de impacto en el pensar o haber intentado emigrar. Esto señalaría la gravedad del impacto emocional de la agresión en el contexto social más íntimo, que para los menores debería de ser el que más seguridad y soporte emocional brindara para un desarrollo sano. Este daño psicológico ha sido corroborado en un metaanálisis 11 que explora en parte la asociación entre exposición a violencia en el hogar con internalización de síntomas, encontrando que los mayores daños emocionales se manifiestan en quiénes habían sido víctimas directas de violencia en el hogar, en comparación con quiénes sólo la testificaron. Los resultados de este estudio coinciden también con otro análisis de mediación ${ }^{42}$, realizado igualmente con el método de Baron \& Kenny ${ }^{39}$, que encuentra en población adolescente que la depresión actúa como una variable que incrementa comportamientos de riesgo, como sexo no protegido, comportamiento violento, ideación suicida, con haber sido víctima de violencia en la familia. Según explican otros investigadores 43 , el impacto de la violencia familiar se produce a nivel neurológico y fisiológico alterando funciones cognitivas, emocionales y sociales, desarrollando trastornos diversos que motivan a los menores a actuar para atenuar sus efectos. El consumo de sustancias, es un recurso para reducir sintomatología depresiva, ansiedad, etc. Y la huida del hogar una forma de cortar la exposición a la violencia. Una de las recomendaciones derivadas de estos resultados sería intervenir contra el uso del castigo corporal en la familia, como modo de corrección del comportamiento 2, ya que es una pauta permisiva de agresión que la modela y justifica por lo que puede escalar hacia castigos físicos de mayor severidad. A su vez, en este estudio la testificación de violencia entre padres, no 
Diferencias por sexo en victimización por violencia y en variables mediadoras

\begin{tabular}{|c|c|c|c|}
\hline & $\begin{array}{c}\text { Total } \\
(\mathrm{N}=13.198) \\
\text { Media }\end{array}$ & $\begin{array}{c}\text { Mujeres } \\
(n=7.266) \\
\text { Media }\end{array}$ & $\begin{array}{c}\text { Hombres } \\
(\mathrm{n}=5.932) \\
\text { Media }\end{array}$ \\
\hline \multicolumn{4}{|l|}{ Entorno familiar } \\
\hline \multicolumn{4}{|l|}{ Víctima de violencia en el hogar } \\
\hline Violencia moderada * & 1,45 & 1,47 & 1,42 \\
\hline Violencia severa * & 1,03 & 1,02 & 1,04 \\
\hline Índice de violencia del padre contra la madre * & 1,66 & 1,76 & 1,54 \\
\hline Índice de violencia de la madre contra el padre * & 1,03 & 1,72 & 1,53 \\
\hline \multicolumn{4}{|l|}{ Ambiente escolar } \\
\hline \multicolumn{4}{|l|}{ Víctima de bullying } \\
\hline Amenazas * & 1,03 & 1,02 & 1,05 \\
\hline Daños a la propiedad * & 1,23 & 1,19 & 1,28 \\
\hline Rechazo social ** & 1,30 & 1,28 & 1,32 \\
\hline Índice de peligro en la escuela $* \star *$ & 1,67 & 1,61 & 1,74 \\
\hline \multicolumn{4}{|l|}{ Vecindario } \\
\hline Índice de violencia en el barrio ** & 2,31 & 2,27 & 2,35 \\
\hline \multicolumn{4}{|l|}{ Variables mediadoras } \\
\hline Autoestima emocional * & 2,19 & 2,27 & 2,08 \\
\hline Autoestima escolar * & 2,89 & 2,97 & 2,80 \\
\hline Depresión * & 2,00 & 2,08 & 1,90 \\
\hline Ideación suicida * & 1,02 & 1,21 & 0,78 \\
\hline Intento suicida * & 0,22 & 0,29 & 0,14 \\
\hline
\end{tabular}

$\star \mathrm{p}<0,001$;

$* * p<0,05$;

$* * * \mathrm{p}<0,01$.

Nota: medias ponderadas, de acuerdo con el diseño por conglomerados.

encontró asociación con pensar o intentar emigrar, siendo una variable relacionada con traumas y comportamientos problemáticos en otros estudios 12 .

En cuanto a la victimización por bullying, la ideación suicida en asociación con daños a la propiedad, y la autoestima emocional por el rechazo social, fueron variables mediadoras de pensar o haber intentado migrar en este estudio. Otras investigaciones internacionales también han encontrado que haber sido víctima de violencia escolar es un factor de riesgo de ideación e intento suicidas 44. Estos síntomas expresan que ha habido un daño en la autoestima, afectando la capacidad para afrontar y resolver situaciones estresantes 45 . El bajo rendimiento académico y la deserción escolar pueden ser consecuencias 46 que llevan al bajo nivel educativo, con mayores dificultades para obtener empleo o empleos con salarios más bajos, por lo que la migración internacional puede ser una opción compensatoria para buscar mayores ingresos. En México ya se está trabajando en programas para reducir los niveles de bullying, pero aún de modo regional. Es importante mejorar la calidad de estas intervenciones en todo el país para reducir la deserción escolar que pudiera generar migración ilegal adolescente.

Por otra parte, las diferencias por sexo encontradas en este estudio muestran que si bien ser hombre es un factor de riesgo de intención migratoria, la mujer posee mayor incremento del riesgo por impacto de variables mediadoras, en las que además presenta mayor nivel de afectación que en hombres. Esto coincide con estudios que señalan que la mujer experimenta los eventos traumáticos más negativamente, con consecuencias en desórdenes mentales mayores 47 . También experimenta más situaciones estresantes de violencia, con menor apoyo y recursos para afrontarlas 48 , lo que se puede explicar desde una perspectiva cultural, que propone que en la población latina coexisten valores 
Tabla 4

Factores de violencias asociados con pensar o haber intentado emigrar a los Estados Unidos por sexo.

\begin{tabular}{|c|c|c|c|c|c|c|}
\hline \multirow[t]{2}{*}{ Variable } & \multicolumn{2}{|c|}{$\begin{array}{c}\text { Total } \\
(\mathrm{N}=13.198)\end{array}$} & \multicolumn{2}{|c|}{$\begin{array}{c}\text { Mujeres } \\
(n=7.266)\end{array}$} & \multicolumn{2}{|c|}{$\begin{array}{l}\text { Hombres } \\
(n=5.932)\end{array}$} \\
\hline & RM & IC95\% & RM & IC95\% & $\mathbf{R M}$ & IC95\% \\
\hline \multicolumn{7}{|l|}{ Sexo } \\
\hline Mujer & 1,00 & & & & & \\
\hline Hombre & 1,88 & 1,$72 ; 2,05$ * & & & & \\
\hline Edad & 1,26 & 1,$16 ; 1,36$ * & 1,27 & 1,$20 ; 1,36$ * & 1,22 & 1,$15 ; 1,29$ * \\
\hline \multicolumn{7}{|l|}{ Nivel socioeconómico } \\
\hline Alto & 1,00 & & 1,00 & & 1,00 & \\
\hline Regular & 0,93 & 0,$77 ; 1,21$ & 1,11 & 0,$89 ; 1,39$ & 0,92 & 0,$76 ; 1,12$ \\
\hline Bajo & 0,94 & 0,$75 ; 1,16$ & 1,15 & 0,$92 ; 1,43$ & 1,02 & 0,$84 ; 1,24$ \\
\hline \multicolumn{7}{|l|}{ Nivel de escolaridad del padre } \\
\hline Alto & 1,00 & & 1,00 & & 1,00 & \\
\hline Regular & 1,10 & 0,$94 ; 1,29$ & 1,06 & 0,$89 ; 1,25$ & 1,11 & 0,$94 ; 1,30$ \\
\hline Bajo & 1,18 & 1,$05 ; 1,32 * \star$ & 1,11 & 0,$92 ; 1,35$ & 1,17 & 0,$98 ; 1,41$ \\
\hline \multicolumn{7}{|l|}{ Nivel de escolaridad de lamadre } \\
\hline Alto & 1,00 & & 1,00 & & 1,00 & \\
\hline Regular & 1,11 & 0,$88 ; 1,41$ & 1,08 & 0,$90 ; 1,28$ & 1,11 & 0,$94 ; 1,31$ \\
\hline Bajo & 1,45 & 1,$08 ; 1,94 * \star \star$ & 1,11 & 0,$91 ; 1,35$ & 1,43 & 1,$18 ; 1,72$ * \\
\hline \multicolumn{7}{|l|}{ Víctima de la violencia en el hogar } \\
\hline Violencia moderada & 1,44 & 1,$28 ; 1,62$ * & 1,49 & 1,$32 ; 1,69 *$ & 1,36 & 1,$19 ; 1,55$ * \\
\hline Violencia severa & 1,12 & 0,$91 ; 1,37$ & 1,55 & 0,$94 ; 2,57$ & 1,12 & 0,$82 ; 1,53$ \\
\hline Índice de violencia del padre contra la madre & 1,06 & 0,$91 ; 1,23$ & 1,03 & 0,$92 ; 1,15$ & 1,04 & 0,$91 ; 1,18$ \\
\hline Violencia de la madre contra el padre & 0,97 & 0,$83 ; 1,14$ & 0,99 & 0,$88 ; 1,11$ & 0,99 & 0,$86 ; 1,12$ \\
\hline \multicolumn{7}{|l|}{ Víctima de bullying } \\
\hline Amenazas & 0,97 & 0,$65 ; 1,42$ & 1,21 & 0,$73 ; 2,01$ & 0,94 & 0,$70 ; 1,25$ \\
\hline Daños a la propiedad & 1,39 & 1,$18 ; 1,65 * \star$ & 1,25 & 1,$02 ; 1,52 * \star \star$ & 1,25 & 1,$08 ; 1,45 * \star$ \\
\hline Rechazo social & 1,20 & 1,$02 ; 1,42 * \star \star$ & 1,20 & 1,$01 ; 1,43 * \star \star$ & 1,36 & 1,$17 ; 1,58$ * \\
\hline Índice de peligro en la escuela & 0,96 & 0,$85 ; 1,09$ & 1,16 & 1,$03 ; 1,29 * *$ & 0,99 & 0,$90 ; 1,10$ \\
\hline Índice de violencia en el barrio & 1,09 & 1,$01 ; 1,18 * \star \star$ & 1,05 & 0,$98 ; 1,13$ & 1,10 & 1,$03 ; 1,17$ ** \\
\hline
\end{tabular}

IC95\%: intervalo de confianza del 95\%; RM: razón de momios.

* $\mathrm{p}<0,001$;

** $\mathrm{p}<0,01$;

$\star \star \star ~ p<0,05$.

tradicionales de machismo y marianismo como parte de una cultura familista 49 , dónde la familia ocupa el centro de importancia en la vida social de las personas, estableciendo mayor control y restricciones sobre las mujeres adolescentes, imponiéndoles valores y tareas tradicionales que favorezcan al mantenimiento y reproducción familiar 50. Esto pudiera manifestarse también en relación a la percepción de la violencia entre los padres, la cuál fue mayor en las mujeres en los resultados de este estudio, porque tiene vínculos familiares más estrechos que el hombre. En este contexto, el comportamiento de la mujer es más controlado, como en el ejercicio sexual y de noviazgo 51, exponiéndola a mayor estrés y violencia familiar con mayor impacto emocional. Estos hallazgos sugerirían que las investigaciones e intervenciones contra violencia familiar deben analizar el papel de los roles y estereotipos de género al considerar las diferencias entre mujeres y hombres en el impacto de la violencia.

Por otra parte, en este estudio, la violencia en el barrio parece afectar más al hombre que a la mujer y en él es un factor de riesgo directo. La presencia de pandillas y narcotráfico puede representar un riesgo influenciado por estereotipos de género con mayor orientación hacia el hombre como víctima 


\section{Tabla 5}

Porcentaje de contribución de las variables mediadoras con pensar o haber intentado emigrar por sexo.

\begin{tabular}{|c|c|c|c|c|c|c|c|c|c|}
\hline & \multicolumn{3}{|c|}{ Total } & \multicolumn{3}{|c|}{ Mujeres } & \multicolumn{3}{|c|}{ Hombres } \\
\hline & RM & IC95\% & $\begin{array}{l}\text { Porcentaje de } \\
\text { impacto }\end{array}$ & RM & IC95\% & $\begin{array}{l}\text { Porcentaje de } \\
\text { impacto }\end{array}$ & RM & IC95\% & $\begin{array}{c}\text { Porcentaje de } \\
\text { impacto }\end{array}$ \\
\hline \multicolumn{10}{|l|}{$\begin{array}{l}\text { moderada en la } \\
\text { familia }\end{array}$} \\
\hline \multicolumn{10}{|l|}{$\begin{array}{l}\text { Variable } \\
\text { mediadora }\end{array}$} \\
\hline $\begin{array}{l}\text { Autoestima } \\
\text { emocional }\end{array}$ & 1,43 & 1,$29 ; 1,60$ & 6,11 & 1,48 & 1,$31 ; 1,69$ ** & 5,80 & 1,35 & 1,$19 ; 1,55$ & 4,9 \\
\hline $\begin{array}{l}\text { Autoestima } \\
\text { escolar }\end{array}$ & 1,43 & 1,$27 ; 1,63^{* *}$ & 3,42 & 1,48 & 1,$30 ; 1,67^{* *}$ & 5,40 & & & \\
\hline Depresión & 1,31 & 1,$17 ; 1,46$ ** & 19,43 & 1,32 & 1,$16 ; 1,50$ ** & 23,40 & 1,29 & 1,$13 ; 1,48$ ** & 12,9 \\
\hline Ideación suicida & 1,29 & 1,$13 ; 1,47$ \# & 36,11 & 1,34 & 1,18; 1,52\# & 36,60 & 1,22 & 1,$06 ; 1,40 \#$ & 34,5 \\
\hline Intento suicida & 1,34 & 1,$18 ; 1,53$ * & 17,77 & 1,36 & 1,$19 ; 1,55^{* *}$ & 21,70 & 1,32 & 1,$16 ; 1,51$ ** & 9,50 \\
\hline \multicolumn{10}{|l|}{$\begin{array}{l}\text { Exposición a rechazo } \\
\text { social en la escuela }\end{array}$} \\
\hline $\begin{array}{l}\text { Modelo inicial * } \\
\text { Modelo final *** } \\
\text { Variable } \\
\text { mediadora }\end{array}$ & \multicolumn{2}{|c|}{$\begin{array}{l}\text { Variable } \\
\text { mediadora }\end{array}$} & & & 1,$01 ; 1,43 \# \#$ & & 1,36 & 1,$17 ; 1,58^{* *}$ & \\
\hline $\begin{array}{l}\text { Autoestima } \\
\text { emocional }\end{array}$ & 1,19 & 1,$01 ; 1,41 \# \#$ & 17,50 & 1,19 & 1,01-1,42 \#\# & 16,90 & 1,35 & 1,$16 ; 1,57^{\text {** }}$ & 13,70 \\
\hline \multicolumn{10}{|l|}{$\begin{array}{l}\text { Exposición a daños } \\
\text { materiales en la } \\
\text { escuela }\end{array}$} \\
\hline $\begin{array}{l}\text { Modelo inicial * } \\
\text { Modelo final *** } \\
\text { Variable medidor }\end{array}$ & 1,39 & 1,$81 ; 1,65$ \# & & 1,25 & 1,02; 1,52 \#\# & & 1,25 & 1,$08 ; 1,45 \#$ & \\
\hline Ideación suicida & 1,37 & 1,$13 ; 1,66 \#$ & 8,10 & 1,22 & 1,$01 ; 1,49 \# \#$ & 8,00 & 1,23 & 1,$12 ; 1,52 \#$ & 7,70 \\
\hline
\end{tabular}

IC95\%: intervalo de confianza del 95\%; RM: razón de momios.

* Riesgo de los adolescentes de pensar o haber intentado emigrar por exposición al tipo de violencia, sin ajuste de mediadoras;

$\star * \mathrm{p}<0,001$;

*** Riesgo de los adolescentes de pensar o haber intentado emigrar por exposición al tipo de violencia, ajustado por las variables mediadoras;

$\# p<0,01$;

$\#$ \# $<0,05$.

de violencia física; sin embargo, no se encontraron variables mediadoras de salud mental relacionadas, a pesar de que el 34,8\% de los jóvenes entre 12 y 17 años de edad sienten miedo y ansiedad de ser víctimas de robo en México 41. Internacionalmente se reporta al estrés, miedo y angustia, variables no evaluadas en este estudio, en asociación con la percepción de violencia en el barrio 52. También se ha señalado que entre la percepción de los niveles de violencia y la victimización hay variables mediadoras como comportamientos evitativos, red social de apoyo y actitudes que impiden afectación psicológica 53. Eso puede explicar que los niveles de victimización por esta violencia no impacten emocionalmente con mayor severidad; pero sería importante evaluar también el miedo y estrés como variables mediadoras en otras investigaciones. 
Por otra parte, este estudio tiene limitaciones. Primero, el diseño no permite establecer causalidad, sino únicamente asociación entre variables evaluadas, y la previsible evolución de estos comportamientos adolescentes es desconocida. Segundo, la información es de carácter retrospectivo, puede crear sesgos debidos a imprecisiones en el recuerdo de la victimización. En tercero, los resultados están restringidos a una población de estudiantes que no representan a todos los adolescentes en México. Aquellos estudiantes no representados en este estudio pudieran tener motivos diferentes para la emigración. En cuarto, existen otros factores, no sólo la violencia mediada por la salud mental, que motivan a los adolescentes a emigrar, desde la reunificación de la familia, efectos del cambio climático hasta crisis económicas. Quinto, existen otras variables mediadoras de salud mental aquí no evaluadas como el estrés y el miedo que pudieran mediar el riesgo.

\section{Conclusión}

Los hallazgos del presente estudio demuestran que, en la población de adolescentes estudiantes mexicanos de nivel medio superior, existe un efecto mediador de las variables de autoestima emocional, autoestima escolar, depresión, ideación e intento suicidas en el riesgo de pensar o haber intentado emigrar a los EE.UU. por victimización de violencia moderada en el hogar. También se ha encontrado un efecto mediador de la autoestima emocional y la ideación suicida por rechazo social y daños a la propiedad en la escuela, respectivamente.

Los hallazgos sugieren crear intervenciones con un enfoque de salud pública para disminuir la violencia en la familia, escuela y barrio, incorporando atención a la salud mental, mejorando la autoestima, y desarrollando competencias en los adolescentes para enfrentar situaciones estresantes, incidiendo en disminuir la depresión, mediante programas de prevención y atención a víctimas, incorporando a los diferentes actores de los entornos sociales descritos para la toma de decisiones en diversos niveles, para que los adolescentes no interrumpan su proyecto académico y de vida, arriesgándose a la migración ilegal internacional. También es importante realizar más investigaciones que exploren la asociación entre exposición a violencias, con la salud mental y emigración internacional en adolescentes latinoamericanos.

Para la salud pública estos hallazgos son importantes, porque señalan la necesidad de dar más importancia a la prevención de la violencia e incorporar la salud mental en la atención de la misma, así como en el desarrollo de políticas públicas para elaboración de programas de intervención, dirigidos a poblaciones móviles en estaciones de tránsito fronterizo, como se ha hecho en relación a la prevención del VIH en diversos países 54, brindando apoyo, consejería y atención a la salud física y mental con migrantes indocumentados.

\section{Colaboradores}

R. Chavez-Ayala participó en la concepción del proyecto, modelamiento estadístico, interpretación, discusión de los resultados y redacción del artículo. E. Orozco-Núñez participó en la concepción del proyecto e interpretación de los datos, y realizó la revisión crítica del contenido. M. Sánchez-Estrada orientó la concepción del artículo, interpretación y discusión de los resultados y realizó comentarios críticos. C. Hernández-Girón colaboró en la literatura y edición de texto, y realizó la revisión crítica del contenido.

\section{Agradecimientos}

Se agradece a la maestra Angélica Rocío Ángeles Llerenas su asesoría en estadística. 


\section{Referencias}

1. WHO Global Consultation on Violence and Health. Violence: a public health priority. Geneva: World Health Organization; 1996. (Document WHO/EHA/SPI.POA.2).

2. Pinheiro PS. Informe mundial sobre violencia contra los niños y las niñas. Geneva: Organización de las Naciones Unidas; 2006.

3. Margolin G, Gordis EB. The effects of family and community violence on children. Annu Rev Psychol 2000; 51:445-79.

4. Salzinger S, Feldman RS, Stockmmer T, Hood J. An ecological framework for understanding risk for exposure to community violence and the effects of exposure on children and adolescents. Aggress Violent Behav 2002; 7:423-51.

5. Gámez-Guadix M, Almendros C. Witnessing interparental violence, parenting practices, and children's long-term psychological distress. Psychosocial Intervention 2011; 20:121-30.

6. Silva AMVL, Taquette SR, Hasselmann MH. Family violence and body mass index among adolescents enrolled in the Bolsa Família Program and treated at a primary care clinic. $\mathrm{Cad}$ Saúde Pública 2014; 30:645-55.

7. Downs WR, Harrison L. Childhood maltreatment and the risk of substance problems in later life. Health Soc Care Community 1998; 6:35-46.

8. Oropeza TR, Caso-López AC, Ayala VH. Relación entre la severidad en el nivel de dependencia al alcohol y la percepción del ambiente familiar. Revista de Psicología Social y Personalidad 2001; 17:1-16.

9. Biggam F, Power K. The quality of perceived parenting experienced by a group of Scottish incarcerated young offenders and its relation to psychological distress. J Adolesc 1998; $21: 167-76$

10. Carlson BE. Outcomes of physical abuse and observation of marital violence among adolescents in placement. J Interpers Violence 1991; 6:526-34.

11. Wolf DA, Crooks CV, Lee V, McIntyre-Smith A, Jaffe PG. The effects of children's exposure to domestic violence: a meta-análysis and critique. Clin Child Fam Psychol Rev 2003; 6:171-87.

12. Chan KL, Yan E, Brownridge DA, Tiwari A, Fong DYT. Childhood sexual abuse associated with dating partner violence and suicidal ideation in a representative household sample in Hong Kong. J Interpers Violence 2011; 26:1763-84.

13. Mrug S, Windle M. Prospective effects of violence exposure across multiple contexts on early adolescents' internalizing and externalizing problems. J Child Psychol Psychiatry 2010; 51:953-61.

14. Hyde J. From home to street: understanding young people's transitions into homelessness. J Adolesc 2005; 28:171-83.
15. Nieri T, Hoffman S, Marsiglia FF, Kulis SS. Interpersonal violence and its association with US migration desires and plans among youths in Guanajuato, Mexico. J Int Migr Integr 2012; 13:365-81.

16. Organisation for Economic Co-operation and Development. TALIS 2013 results: an international perspective on teaching and learning. http://www.oecd-ilibrary.org/education/talis2013-results_9789264196261-en (accedido el 10/Sep/2015).

17. Hawker DSJ, Boulton MJ. Twenty years' research on peer victimization and psychosocial maladjustment: a meta-analytic review of cross-sectional studies. J Child Psychol Psychiatry 2000; 41:441-55.

18. Rigby K. Consequences of bullying in schools. Can J Psychiatry 2003; 48:583-90.

19. Esbensen FA, Carson DC. Consequences of being bullied: results from a longitudinal assessment of bullying victimization in a multisite sample of American students. Youth Soc 2009; 41:209-33

20. Secretaría de Salud. Informe Nacional sobre violencia y salud. México DF: Secretaría de Salud; 2006.

21. Oficina del Alto Comisionado de las Naciones Unidas para los Refugiados. Arrancados de raíz. Causas que originan el desplazamiento transfronterizo de niños, niñas y adolescentes no acompañados y/o separados de Centroa mérica y su necesidad de protección internacional. http://www.acnur.org/t3/fileadmin/ scripts/doc.php?file $=\mathrm{t} 3 /$ fileadmin $/$ Documen tos/Publicaciones/2014/9828 (accedido el 12/ Sep/2015)

22. De Alba F, Castillo MA, Verduzco G. Migraciones internacionales: los grandes problemas de México. México DF: El Colegio de México; 2010.

23. Andrews TJ, Ybarra VD, Miramontes T. Negotiating survival: undocumented Mexican immigrant women in the Pacific Northwest. Soc Sci J 2002; 39:431-49.

24. Volkmann T, Shin SS, Garfein RS, Patterson TL, Pollini RA, Wagner KD, et al. Border crossing to inject drugs in Mexico among injection drug users in San Diego, California. J Immigrant Minority Health 2012; 14:281-6.

25. Borges G, Rafful C, Benjet C, Tancredi DJ, Saito N, Aguilar-Gaxiola S, et al. Mexican immigration to the US and alcohol and drug use opportunities: does it make a difference in alcohol and/or drug use? Drug Alcohol Depend 2012; 125 Suppl 1:S4-11.

26. Simons R, Whitbeck L. Running away during adolescence as a precursor to adult homelessness. Soc Serv Rev 1991; 65:225-47. 
27. Sowell RL, Holtz CS, Velázquez G. HIV infection returning to Mexico with migrant workers: an exploratory study. J Assoc Nurses AIDS Care 2008; 19:267-82.

28. Familiar I, Borges G, Orozco R, Medina-Mora ME. Mexican migration experiences to the US and risk for anxiety and depressive symptoms. J Affect Disord 2011; 130:83-91.

29. Hanson GH, Spilimbergo A. Illegal immigration, border enforcement, and relative wages: evidence from apprehensions at the U.S.-Mexico border. Am Econ Rev 1999; 89:1337-57.

30. Orrenius PM. The role of family networks, coyote prices and the rural economy in migration from Western Mexico: 1965-1994. Dallas: Federal Reserve Bank of Dallas; 1999. (Working Paper, 9910).

31. Instituto Nacional de Estadística y Geografía e Informática. Principales resultados del Censo de Población y Vivienda. México DF: Instituto Nacional de Estadística y Geografía e Informática; 2010.

32. Bronfman M, Guiscafré H, Castro V, Castro R, Gutiérrez G. La medición de la desigualdad: una estrategia metodológica, análisis de las características socioeconómicas de la muestra. Arch Invest Med Méx 1988; 19:351-60.

33. Straus MA, Hamby SL, Finkelhor D, Moore DW, Runyan D. Identification of child maltreatment with the Parent-Child Conflict Tactics Scales: development and psychometric data for a national sample of American parents. Child Abuse Negl 1998; 22:249-70.

34. Díaz-Aguado MJ, Martínez-Arias R, MartínSeoane G. Prevención de la violencia y lucha contra la exclusión desde la adolescencia. v. 1. La violencia entre iguales en la escuela y en el ocio: estudios comparativos e instrumentos de evaluación. Madrid: Instituto de la Juventud; 2004.

35. Musitu G, García F, Gutiérrez M. AFA: scale of self-concept form A. 2a Ed. Madrid: TEA Ediciones; 1994.

36. Coopersmith $\mathrm{S}$. The antecedents of the selfesteem. San Francisco: W. H. Freeman; 1967.

37. González-Forteza C, Wagner FA, Jiménez A. Escala de Depresión del Centro de Estudios Epidemiológicos (CES-D) en México: análisis bibliométrico. Salud Mental 2012; 35:13-20.

38. Borges G, Orozco R, Medina-Mora ME. Risk index for attempted suicide in Mexico. Salud Pública Mex 2012; 54:595-606.

39. Baron RM, Kenny DA. The moderator-mediator variable distinction in social psychological research: conceptual, strategic and statistical considerations. J Pers Soc Psychol 1986; 51:1173-82.

40. Kenny DA. Mediation. http://davidakenny.net (acceedido el 06/Jan/2016).

41. Instituto Mexicano de la Juventud; Consejo Nacional para Prevenir la Discriminación. Encuesta Nacional sobre Discriminación en México. Resultados sobre las y los jóvenes. http://www.conapred.org.mx/userfiles/files/ Enadis-JOVENES-Accss.pdf (accedido el 15/ $\mathrm{Jul} / 2015)$.
42. Okello J, Nakimuli-Mpungu E, Musisi S, Broekaert E, Derluyn I. War-related trauma exposure and multiple risk behaviors among school-going adolescents in Northern Uganda: the mediating role of depression symptoms. J Affect Disord 2013; 151:715-21.

43. De Bellis MD. Developmental traumatology: the psychobiological development of maltreated children and its implications for research, treatment, and policy. Dev Psychopathol 2001; 13:539-64.

44. Geel MV, Vedder P, Tanilon J. Relationship between peer victimization, cyberbullying, and suicide in children and adolescents: a metaanalysis. JAMA Pediatr 2014;168:435-42.

45. Birndorf SDO, Ryan S, Auinger P, Aten ARN. High self-steem among adolescents: longitudinal trends, sex differences, and protective factors. J Adolesc Health 2005; 37:194-201.

46. Esbensen FA, Carson DC. Consequences of being bullied: results from a longitudinal assessment of bullying victimization in a multisite sample of American students. Youth Soc 2009; 41:209-33.

47. Kucharska J. Sex differences in the appraisal of traumatic events and psychopatology. Psychol Trauma 2016; Epub ahead of print.

48. Springer KW, Sheridan J, Kuo D, Carnes M. Long-term physical and mental health consequences of childhood physical abuse: results from a large population-based sample of men and women. Child Abuse Negl 2007; 31: 517-30.

49. Cauce AM, Domenech-Rodriguez M. Latino families: myths and realities. In: Contreras JM, Kerns KA, Neal-Barnett AM, editors. Latino children and families in the United States: current research and future directions. Westport: Praeger; 2002. p. 3-25.

50. Azmitia A, Brown JR. Latino immigrant parents' beliefs about the "path of life" of their adolescent children. In: Contreras JM, Kerns KA, Neal-Barnett AM, editors. Latino children and families in the United States. Westport: Praeger; 2002. p. 77-106.

51. Raffaelli M, Ontai LL. Gender socialization in Latino/a families: results from two retrospective studies. Sex Roles 2004; 50:287-99.

52. Goldman-Mellor S, Margerison-Zilko C, Allen K, Cerda M. Perceived and objectively-measured neighborhood violence and adolescent psychological distress. J Urban Health 2016; 93:758-69.

53. Lorenc T, Clayton S, Neary D, Whitehead M, Petticrew M, Thomson H, et al. Crime, fear of crime, environment, and mental health and well being: mapping review of theories and causal pathways. Health Place 2012; 18 : 757-65.

54. Leyva R, Caballero M, Bronfman M. Respuesta social ante la movilidad poblacional y el VIH/ SIDA: experiencias en Centroamérica y México. Cuernavaca: Instituto Nacional de Salud Pública; 2005. 


\section{Abstract}

The aim of this study was to estimate the role of victimization by violence among Mexican adolescents that have considered or attempted migrating to the United States, including mental health variables (emotional self-esteem, self-esteem in school, depression, suicidal ideation, and attempted suicide) as mediators of the effects. The study used a cross-sectional design with a stratified cluster sample of 13,198 adolescents from the $2^{\text {nd }}$ Mexican National Survey on Exclusion, Intolerance, and Violence in public schools in 2009. The analysis used the regression models proposed by Baron es Kenny. Prevalence of having considered or attempted cross-border migration was 23.1\%. Mean age was 16.36 years. Female adolescents constituted $54.9 \%$ of the sample, and $56 \%$ were lower-income. Mental health variables that acted as partial mediators were suicidal ideation (35.9\%), depression (19.2\%), attempted suicide (17.7\%), emotional self-esteem (6.2\%), and self-esteem in school (3.4\%) for moderate family violence, and emotional self-esteem (17.5\%) for social rejection in school and suicidal ideation (8.1\%) for physical harm in school. Female adolescents showed greater impact from mediators than men in considering or having attempted cross-border migration. The study discusses the importance of incorporating the prevention of violence in the social contexts studied here and incorporating mental health in dealing with violence in adolescents and in public health programs in transit areas for illegal migrants.

Domestic Violence; Mental Health; Emigration and Immigration; Adolescent

\section{Resumo}

O objetivo deste estudo foi estimar o papel de vitimização por violência entre adolescentes mexicanos que consideraram ou tentaram migrar para os Estados Unidos, incluindo variáveis de saúde mental (autoestima emocional, autoestima na escola, depressão, ideação suicida e tentativa de suicídio) como mediadores dos efeitos. O estudo utilizou um desenho transversal com uma amostra estratificada por conglomerados de 13.198 adolescentes da 2a Pesquisa Nacional sobre Exclusão, Intolerância e Violência nas escolas públicas mexicanas em 2009. A análise utilizou os modelos de regressão proposto por Baron er Kenny. A prevalência de ter considerado ou tentado migração internacional foi de 23,1\%. A idade média foi de 16,36 anos. Adolescentes do sexo feminino constituíram 54,9\% da amostra e 56\% eram de classe socioeconômica baixa. Variáveis de saúde mental que atuavam como mediadores parciais foram: ideação suicida (35,9\%), depressão (19,2\%), tentativa de suicídio (17,7\%), autoestima emocional $(6,2 \%)$ e autoestima na escola $(3,4 \%)$ para violência familiar moderada, autoestima emocional (17,5\%) para a rejeição social na escola e ideação suicida $(8,1 \%)$ para danos físicos na escola. Encontrou-se maior impacto de mediadores entre adolescentes femininas do que em adolescentes masculinos em pensar ou ter tentado emigrar. $O$ estudo discute a importância de incorporar a prevenção da violência e a saúde mental nos contextos sociais estudados para lidar com a violência em adolescentes e em programas de saúde pública em áreas de trânsito para migrantes ilegais.

Violência Doméstica; Saúde Mental; Emigração e Imigração; Adolescente
Recibido el 10/Jul/2016

Versión presentada el 06/Ene/2017

Aprobado el 06/feb/2017 